\title{
Initializing the EM Algorithm for Univariate Gaussian, Multi-Component, Heteroscedastic Mixture Models by Dynamic Programming Partitions
}

\author{
Andrzej Polanski*, ${ }^{*}$, Michal Marczyk ${ }^{\dagger,}$, Monika Pietrowska ${ }^{\ddagger, \|}$, \\ Piotr Widlak ${ }^{\ddagger, * *}$ and Joanna Polanska ${ }^{\dagger, \dagger \dagger}$ \\ *Institute of Informatics, Silesian University of Technology \\ 44-100 Gliwice, Poland \\ ${ }^{\dagger}$ Data Mining Group, Institute of Automatic Control \\ Silesian University of Technology \\ 44-100 Gliwice, Poland \\ ${ }^{\ddagger}$ Maria Sklodowska-Curie Memorial Cancer Center \\ and Institute of Oncology, Branch in Gliwice \\ 44-101 Gliwice, Poland \\ §andrzej.polanski@polsl.pl \\ \michal.marczyk@polsl.pl \\ " \\ **widlak@io.gliwice.pl \\ ${ }^{\dagger \dagger}$ joanna.polanska@polsl.pl \\ Received 4 August 2015 \\ Revised 1 May 2017 \\ Accepted 11 May 2017 \\ Published 3 July 2017
}

\begin{abstract}
Setting initial values of parameters of mixture distributions estimated by using the EM recursive algorithm is very important to the overall quality of estimation. None of the existing methods are suitable for heteroscedastic mixtures with a large number of components. We present relevant novel methodology of estimating the initial values of parameters of univariate, heteroscedastic Gaussian mixtures, on the basis of dynamic programming partitioning of the range of observations into bins. We evaluate variants of the dynamic programming method corresponding to different scoring functions for partitioning. We demonstrate the superior efficiency of the proposed method compared to existing techniques for both simulated and real datasets.
\end{abstract}

Keywords: Gaussian mixtures; EM algorithm; dynamic programming; mass spectra.

${ }^{\dagger \dagger}$ Corresponding author.

This is an Open Access article published by World Scientific Publishing Company. It is distributed under the terms of the Creative Commons Attribution 4.0 (CC-BY) License. Further distribution of this work is permitted, provided the original work is properly cited. 


\section{A. Polanski et al.}

\section{Introduction}

A problem of crucial importance in applications of the expectation maximization (EM) recursive algorithm [McLachlan and Peel (2000)] for fitting normal mixture models to data is the choice of initial values for mixture parameters. A good choice of the starting point for the EM iterations can result in reducing the probability of erroneous estimation of parameters, along with increasing the odds of achieving EM convergence and potentially improving the time needed to obtain it. Approaches to initializing EM iterations have been extensively discussed and studied McLachlan and Peel (2000); Karlis and Xekalaki (2003); Biernacki et al. (2003); Biernacki (2004); Maitra (2009); O'Hagan et al. (2012); Melnvkov and Melnvkov (2012)]. A simple approach is random initialization involving the generation of initial values of parameters and component weights (mixing proportions) by using assumed probability distributions [McLachlan and Peel (2000)]. Another simple idea is using data quantiles to estimate initial means and variances of components to start the EM iterations. A group of approaches involve using some kind of clustering procedure (hierarchical clustering or $k$-means clustering) applied for the data set to compute initial parameters for EM iterations [Biernacki et al. (2003); Maitra (2009); O'Hagan et al. (2012)]. Some other ideas for computing initial values involve using sample moments of data Karlis and Xekalaki (2003)], a method of using singular value decompositions for multivariate mixtures Melnvkov and Melnvkov (2012)], or using properties of EM trajectories and data moments to limit the search space for initial parameters of multivariable Gaussian mixtures Biernacki (2004)]. Available software packages for mixture modeling McLachlan and Peel (1999); Biernacki et al. (2006); Fraley and Raftery (1999); Richardson and Green (1997)] offer different possibilities for setting initial conditions for EM iterations.

A challenge for the existing methods is the decomposition of univariate, heteroscedastic Gaussian mixtures with a large number of components. It can be verified in practical computations that with the increase of the number of components in the mixture, the application of the EM algorithm making use of published methodology for setting initial conditions would lead to mixture models of the progressively worse quality. Yet, problems of mixture decompositions of univariate models where the numbers of components are large are encountered in many applications. Some examples where numbers of components may range from tens to hundreds are frequency (amplitude or power) spectra of different time domain signals in vibration diagnostics or speech recognition [Eirola and Lendasse (2013)], Fraiha Machado et al. (2013)]; nuclear magnetic resonance Fourier spectra of the free induction decay signals Chylla (2012)]; proteomic mass spectrometry signals Pietrowska et al. (2011); Polanski et al. (2015)] or read counts signals in next generation sequencing data [ibrahim et al. (2015)].

The aim of this study is to develop and evaluate a method for estimating initial values of parameters for EM iterations for univariate, multi-component, heteroscedastic Gaussian mixtures based on dynamic programming partitioning 
algorithm. Partitioning the data points into bins with dynamic programming determines the initial values of weights, means and variances of Gaussian components for the EM algorithm. The idea of partitioning an interval into bins with dynamic programming with the aim of obtaining a solution to some fitting or estimation problem was formulated by Bellman Bellman (1961)] and then studied/used by other authors in many contexts [e.g., Hébrail et al. (2010)]. The advantage of using dynamic programming over partitioning (clustering) heuristics by hierarchical clustering or greedy algorithms is that the dynamic programming method allows for obtaining the global optimal solution for a given quality function. According to the authors' best knowledge, this study constitutes the first documented application of dynamic programming to initalizing EM iterations.

In our paper, we are distinguishing two types of data. The first type is called "classic". In this case, the dataset consists of the list of single measurements. The second type is called "dense bins data". It is the special case where there are numerous multiple measurements. Multiple measurements typically result from limited resolution of the measurement device. Such a case is common in many applications when measurements are frequencies, intensities or counts. e.g., in our real proteomic dataset the measured signals are ion counts coming from ionized particles detector. All such data types are covered by the binned data model McLachlan and Peel [2000, Chap. 9)] which we complement with the additional assumption of dense bins. We explain in detail what we mean by dense bins at the end of Sec. 2

We present a dynamic programming algorithm for computing initial values for mixture parameters for EM iterations for both "classic" and "dense bins data" models. We also study the problem of the choice of the scoring function. We compare several possible scoring functions and, on the basis of computational experiments, we propose the one best suited to the problem of computing initial values of mixture parameters. For a number of artificially generated univariate data sets with multiple Gaussian, heteroscedastic components, we compare established methods of initialization of EM iterations with the proposed algorithm, capturing its improved performance. We also apply the method for initializing EM iterations to the real protein mass spectrometry dataset and we demonstrate the superior efficiency of the proposed method compared to existing techniques.

\section{Gaussian Mixture Modeling}

Gaussian mixture modeling for the classic case of a dataset of univariate scalar observations, $\mathbf{x}=\left[x_{1}, x_{2}, \ldots, x_{N}\right]$ involves estimating the vector of mixture parameters

$$
\mathbf{p}=\left[\alpha_{1}, \ldots, \alpha_{K}, \mu_{1}, \ldots, \mu_{K}, \sigma_{1}, \ldots, \sigma_{K}\right]
$$

(component weights, means and standard deviations) of the mixture probability density function (pdf)

$$
f^{\operatorname{mix}}(x, \mathbf{p})=\sum_{k=1}^{K} \alpha_{k} f\left(x, \mu_{k}, \sigma_{k}\right)
$$




\section{A. Polanski et al.}

where $f\left(x, \mu_{k}, \sigma_{k}\right)$ is the Gaussian pdf, $\sum_{k=1}^{K} \alpha_{k}=1, \alpha_{k}>0$. Parameter estimation is done by maximization of the log-likelihood function McLachlan and Peel (2000)]

$$
L(\mathbf{x}, \mathbf{p})=\sum_{n=1}^{N} \log f^{\operatorname{mix}}\left(x_{n}, \mathbf{p}\right) .
$$

For datasets of the "dense bins" type, measurements are frequencies, numbers of counts or intensities. In this case $\mathbf{x}$ is a vector of centers of bins, which follows from the plan of the performed experiment. e.g., in proteomic spectra $\mathbf{x}$ is a vector of mass-to-charge $(\mathrm{m} / z)$ values, which correspond to expected range of masses of particles registered in the measurement device. The corresponding vector $\mathbf{y}=\left[y_{1}, y_{2}, \ldots, y_{N}\right]$ denotes observations based on $\mathbf{x}$. In the binned data model, McLachlan and Peel (2000), Chap. 9], we assume that observations (counts) $y_{n}, n=1, \ldots, N$ are generated by a multinomial distribution with probabilities $p_{n}$ defined by the areas of bins,

$$
p_{n}=\sum_{k=1}^{K} \alpha_{k}\left[\Phi\left(x_{n}+\frac{\delta}{2}, \mu_{k}, \sigma_{k}\right)-\Phi\left(x_{n}-\frac{\delta}{2}, \mu_{k}, \sigma_{k}\right)\right],
$$

where $\Phi\left(x, \mu_{k}, \sigma_{k}\right)$ is the cumulative probability distribution function of the Gaussian distribution and $\delta$ is the bin width. We assume that centers of bins are equally spaced, but more general situation where centers of bins are non-uniformly spaced can also be easily addressed.

The assumption that bins are "dense", is equivalent to validity of the following approximation

$$
\Phi\left(x_{n}+\frac{\delta}{2}, \mu_{k}, \sigma_{k}\right)-\Phi\left(x_{n}-\frac{\delta}{2}, \mu_{k}, \sigma_{k}\right) \approx \delta f\left(x_{n}, \mu_{k}, \sigma_{k}\right) .
$$

Using the above approximation leads to the log-likelihood function defined (up to the constant dependent only on $\delta$ ) by the following formula:

$$
L(\mathbf{x}, \mathbf{y}, \mathbf{p})=\sum_{n=1}^{N} y_{n} \log f^{\operatorname{mix}}\left(x_{n}, \mathbf{p}\right) .
$$

The assumption of dense bins is satisfied for the proteomic dataset used in this study as well as for all data types mentioned in the papers referenced in Sec. 1 .

\subsection{EM iterations}

The maximization of the log-likelihood functions, either (2) for the "classic" case or (5) for the "dense bins" data case, is done with EM iterations - the successive updates of the parameter vector $\mathbf{p}^{\text {old }} \leftarrow \mathbf{p}^{\text {new }}$, where

$$
\mathbf{p}^{\text {old }}=\left[\alpha_{1}^{\text {old }}, \ldots, \alpha_{K}^{\text {old }}, \mu_{1}^{\text {old }}, \ldots, \mu_{K}^{\text {old }}, \sigma_{1}^{\text {old }}, \ldots, \sigma_{K}^{\text {old }}\right],
$$

and

$$
\mathbf{p}^{\text {new }}=\left[\alpha_{1}^{\text {new }}, \ldots, \alpha_{K}^{\text {new }}, \mu_{1}^{\text {new }}, \ldots, \mu_{K}^{\text {new }}, \sigma_{1}^{\text {new }}, \ldots, \sigma_{K}^{\text {new }}\right]
$$


Formulae for EM iterations for the classic case are given in e.g., McLachlan and Peel 2000 and Bilmes 1998. The version of EM iterations appropriate for binned data with dense bins is very similar to standard EM iterations. It involves defining conditional distributions of hidden (latent) variables, $\chi_{n}$ (corresponding to unknown assignments of observations to components)

$$
P\left[\chi_{n}=k \mid x_{n}\right]=\frac{\alpha_{k}^{\text {old }} f\left(x_{n}, \mu_{k}^{\text {old }}, \sigma_{k}^{\text {old }}\right)}{\sum_{\varkappa=1}^{K} \alpha_{\varkappa}^{\text {old }} f\left(x_{n}, \mu_{\varkappa}^{\text {old }}, \sigma_{\varkappa}^{\text {old }}\right)}
$$

and updates of parameters estimates given by

$$
\begin{aligned}
\alpha_{k}^{\text {new }} & =\frac{\sum_{n=1}^{N} y_{n} P\left[\chi_{n}=k \mid x_{n}\right]}{\sum_{n=1}^{N} y_{n}}, \\
\mu_{k}^{\text {new }} & =\frac{\sum_{n=1}^{N} y_{n} x_{n} P\left[\chi_{n}=k \mid x_{n}\right]}{\sum_{n=1}^{N} y_{n} P\left[\chi_{n}=k \mid x_{n}\right]}, \\
\left(\sigma_{k}^{\text {new }}\right)^{2} & =\frac{\sum_{n=1}^{N} y_{n}\left(x_{n}-\mu_{k}^{\text {new }}\right)^{2} P\left[\chi_{n}=k \mid x_{n}\right]}{\sum_{n=1}^{N} y_{n} P\left[\chi_{n}=k \mid x_{n}\right]},
\end{aligned}
$$

$k=1,2, \ldots, K$.

\subsection{Preventing divergence of EM iterations}

Some assumptions should be made concerning the execution of the EM iterations. In the case of unequal variances of components of the Gaussian mixture, considered here, the log-likelihood (2) or (5) is unbounded [see McLachlan and Peel (2000), Secs. 2.5, 2.8; Kiefer and Wolfowitz (1956)]. The unboundedness results in a possibility of encountering divergence of EM iterations in practical computations and in the need for using approaches for preventing divergence Yao (2010); Ingrassia (2004)]. We prevent divergence of EM iterations by applying simple constraint conditions. Namely, we do not allow the standard deviations of the Gaussian components and mixing proportions to fall below given threshold values $\sigma_{\min }$ and $\alpha_{\min }$ i.e., we augment the equations for iterations for standard deviations and component weights by additional constraints

$$
\sigma_{k}^{\text {new }} \leftarrow \max \left(\sigma_{k}^{\text {new }}, \sigma_{\min }\right)
$$

and

$$
\alpha_{k}^{\text {new }} \leftarrow \max \left(\alpha_{k}^{\text {new }}, \alpha_{\min }\right) .
$$

The above constraints are sufficient to prevent divergence of EM iterations.

The values of $\sigma_{\min }$ and $\alpha_{\min }$ can be reasonably chosen on the basis of some, even rough, knowledge of the possible ranges of parameters of estimated mixtures. The constraint values used in the EM iterations in this study are $\sigma_{\min }=10^{-2}, \alpha_{\min }=$ $10^{-4}$ for the simulated datasets and $\sigma_{\min }=1, \alpha_{\min }=10^{-5}$ for the proteomic dataset. 


\section{A. Polanski et al.}

\section{Problem Formulation}

The problem tackled in this study concerns the determination of initial values for mixture parameters for EM iterations, $\mu_{k}^{\text {ini }}, \sigma_{k}^{\text {ini }}, \alpha_{k}^{\text {ini }}, k=1,2, \ldots, K$, to yield the best quality of the mixture parameter estimates. All methods for setting initial conditions for EM iterations, compared here, rely on partitioning the data range performed according to some criterion. We assume that the observations $x_{1}, x_{2}, \ldots, x_{N}$ are sorted in ascending order

$$
x_{1}<x_{2}<\cdots<x_{N} .
$$

Partitions are defined by blocks $B_{1}, B_{2}, \ldots, B_{K}$. Blocks are contiguous ranges of indices. The $k$ th block containing successive indices $i, i+1, \ldots, j$ can be defined as the set $B_{k}=\{i, i+1, \ldots, j\}$. We also use the term data in the block, meaning either $\left\{x_{i}, x_{i+1}, \ldots, x_{j}\right\}$ or $\left\{x_{i}, y_{i}, x_{i+1}, y_{i+1}, \ldots, x_{j}, y_{j}\right\}$.

Assignment of the range of indices $i, i+1, \ldots, j$ to block $B_{k}$ depends on the applied solution to the partitioning problem. Different methods of partitioning will lead to different assignments.

Partitions defined by blocks are used for computing initial values for parameters. The initial means, standard deviations and mixing proportions, implied by block $B_{k}=\{i, i+1, \ldots, j\}$, are computed as

$$
\begin{aligned}
\mu_{k}^{\text {ini }} & =\frac{1}{j-i+1} \sum_{n=i}^{n=j} x_{n}, \\
\sigma_{k}^{\text {ini }} & =\sqrt{\frac{1}{j-i+1} \sum_{n=i}^{n=j}\left(x_{n}-\mu_{k}^{\text {ini }}\right)^{2}},
\end{aligned}
$$

and

$$
\alpha_{k}^{\text {ini }}=\frac{\# B_{k}}{N}=\frac{j-i+1}{N} .
$$

In the above expression, $\# B_{k}$ denotes the number of indices in the block $B_{k}$.

In order to provide expressions corresponding to (13)-115), for the case of binned data with dense bins, let us first define

$$
w_{n}=\frac{y_{n}}{\sum_{\nu=i}^{\nu=j} y_{\nu}} .
$$

With the above definition the initial values for means, standard deviations and mixing proportions are computed as

$$
\begin{aligned}
\mu_{k}^{\text {ini }} & =\sum_{n=i}^{n=j} x_{n} w_{n}, \\
\sigma_{k}^{\text {ini }} & =\sqrt{\sum_{n=i}^{n=j} w_{n}\left(x_{n}-\mu_{k}^{\text {ini }}\right)^{2}},
\end{aligned}
$$


and

$$
\alpha_{k}^{\text {ini }}=\frac{\sum_{\nu=i}^{\nu=j} y_{\nu}}{\sum_{n=1}^{N} y_{n}} .
$$

\section{Initializing EM Iterations by Using a Dynamic Programming Partitioning Algorithm}

In this section, we describe the algorithm for computing partitions by using a dynamic programming method. Partitioning of the observations involves defining $K$ blocks

$$
B_{1}, B_{2}, \ldots, B_{K}
$$

For each of the blocks (more precisely, for the data in each block), we compute a scoring function denoted by $Q\left(B_{k}\right)$. The problem of optimal partitioning involves defining blocks (20), such that the cumulative scoring index $Q\left(B_{1}, B_{2}, \ldots, B_{K}\right)$ is minimized,

$$
Q\left(B_{1}, B_{2}, \ldots, B_{K}\right)=\sum_{k=1}^{K} Q\left(B_{k}\right) \rightarrow \min .
$$

The solution to the optimal partitioning problem (21) by dynamic programming Bellman (1961)] is obtained by an iterative application of the following Bellman equation

$$
Q_{1 \ldots j}^{\mathrm{opt}}(k+1)=\min _{i=1 \ldots j-1} Q_{1 \ldots i-1}^{\mathrm{opt}}(k)+Q\left(B_{k}\right),
$$

where $Q_{1, \ldots, i}^{\text {opt }}(k)$ denotes the optimal cumulative partial score of partitioning the range $1, \ldots, i$ into $k$ blocks.

\subsection{Scoring functions}

The scoring function $Q\left(B_{k}\right)$ used in the dynamic programming algorithm of data partitioning (21) - (22) should be designed in such a way that it allows detection of dense subgroups in the data. A scoring function often used in the literature is the weighted sum of squares of within block deviations of data points from the mean

$$
Q\left(B_{k}\right)=\sum_{n=i}^{n=j} \gamma_{k}\left[x_{n}-\frac{1}{j-i+1} \sum_{\nu=i}^{\nu=j} x_{\nu}\right]^{2} .
$$

Often, weights $\gamma_{k}$ are assumed as normalizing factors for the number of elements in the block, which leads to the scoring function, which we define as $Q_{1}\left(B_{k}\right)$

$$
Q_{1}\left(B_{k}\right)=\frac{1}{(j-i+1)} \sum_{n=i}^{n=j}\left[x_{n}-\frac{1}{j-i+1} \sum_{\nu=i}^{\nu=j} x_{\nu}\right]^{2} .
$$

The scoring function $Q_{1}\left(B_{k}\right)$ is a within-block variance of the data. Intuitively, $Q_{1}\left(B_{k}\right)$ is a reasonable measure of the concentration of data points within the defined clusters for detecting clusters in the data. 


\section{A. Polanski et al.}

Other definitions of scoring functions are also possible, and the impact of a scoring function on the quality of the data partition, subsequently leading to the estimation of the mixture parameters, is an interesting issue to tackle. Therefore, below we define other scoring indices, obtained by some modifications of $Q_{1}\left(B_{k}\right)$ (24).

The second scoring function $Q_{2}\left(B_{k}\right)$ is a within-block, standard deviation

$$
Q_{2}\left(B_{k}\right)=\sqrt{Q_{1}\left(B_{k}\right)}
$$

We also use a third scoring function $Q_{3}\left(B_{k}\right)$ defined as a ratio of the withinblock, standard deviation and the block sample range,

$$
Q_{3}\left(B_{k}\right)=\frac{\sqrt{Q_{1}\left(B_{k}\right)}}{x_{j}-x_{i}} .
$$

The property of the scoring function $Q_{3}\left(B_{k}\right)$ is that it is dimensionless and, in the case of a large number of data points, depends only on the shape of the probability distribution function of the data.

Finally, we also introduce a fourth scoring function, $Q_{4}\left(B_{k}, \Delta\right)$, which is a modification of the scoring function $Q_{3}\left(B_{k}\right)$, such that some preference is given to wider blocks in comparison to narrower ones. The idea of giving preference to wider blocks is motivated by the fact that very narrow blocks detected by $Q_{3}\left(B_{k}\right)$ may correspond to random variations of the data rather than to the true dense subgroups related to the structure of the Gaussian components. In order to give some preference to wider blocks we modify $Q_{3}\left(B_{k}\right)$ by introducing an additional parameter $\Delta$, which leads to the scoring function $Q_{4}\left(B_{k}, \Delta\right)$

$$
Q_{4}\left(B_{k}, \Delta\right)=\frac{\Delta+\sqrt{Q_{1}\left(B_{k}\right)}}{x_{j}-x_{i}} .
$$

Adding a positive constant $\Delta$ in the numerator of the above expression results in limiting the possibility of "shrinking" the numerator of $Q_{4}\left(B_{k}, \Delta\right)$ to values very close to 0 , which can happen when narrow random "peaks" occur in the data.

\subsection{Scoring functions for binned data}

When applying the dynamic programming method to binned data, the corresponding expression for the scoring function $Q_{1}$ is

$$
Q_{1}\left(B_{k}\right)=\sum_{n=i}^{n=j} w_{n}\left(x_{n}-\sum_{\nu=i}^{\nu=j} x_{\nu} w_{\nu}\right)^{2},
$$

where $w_{n}$ is defined as in (16). The formulas for scoring functions $Q_{2}, Q_{3}$ and $Q_{4}$, for binned data, are given by Eqs. (25)-(27), with (24) replaced by Eq. (28).

We are using the same notation for scoring functions $Q_{1}-Q_{4}$ for both cases of classic and binned data types, but it is always clear from the contexts, which case is considered. 


\subsection{Properties of different scoring functions}

Different scoring indices may lead to different partitions of the data. Therefore, in the following computational experiments we apply and compare all of the proposed scoring indices $Q_{1}\left(B_{k}\right), Q_{2}\left(B_{k}\right), Q_{3}\left(B_{k}\right)$ and $Q_{4}\left(B_{k}, \Delta\right)$. Some preliminary observations (later systematically verified) are summarized below.

The index $Q_{1}\left(B_{k}\right)$ has a tendency to over-penalize wide components, which can result in splitting some of the wider components in two and in merging narrow components into one. The index $Q_{2}\left(B_{k}\right)$ shows high sensitivity for cases where there is little overlap between components. However, when the overlap between components increases, it has the tendency to randomly merge components. The index $Q_{3}\left(B_{k}\right)$ shows advantages following from its dimensionless construction and often leads to correct partitions. However, it shows sensitivity to noise in the data. Finally, the modified index $Q_{4}\left(B_{k}, \Delta\right)$ allows improving the performance of $Q_{3}\left(B_{k}\right)$ by increasing robustness against noise in the data.

\section{Reference Methods of Setting Initial Condition for EM Iterations}

We compare the dynamic programming partitions to several reference methods for setting initial conditions for EM iterations. These methods were already studied in the literature Biernacki et al. (2003); Maitra (2009); Fralev and Rafterv (1999)] and they were proven to be useful approaches for estimating mixture decompositions of datasets. The first examined algorithm is the method of equal quantiles, which is the default option in the software package Mclust [Fraley and Raftery (1999)]. The bins are defined by equal quantiles of the dataset. Two other reference methods are hierarchical clustering algorithms [Hastie et al. (2009)], where clusters of samples are created by successive merging based on the distances between samples and/or the distances between clusters of samples. We apply two versions of hierarchical clustering, with average and complete linkage [Hastie et al. (2009)]. Initial values for component means, standard deviations and weights are defined by blocks obtained by the application of hierarchical clustering algorithms.

\section{Results}

We have conducted several computational experiments for systematic comparisons of the methods of setting initial values of parameters for EM iterations by the dynamic programming algorithm and the three reference methods. We are using the following abbreviations for algorithms for setting initial conditions: E-Q equal quantiles algorithm, H-clu-c — hierarchical clustering algorithm with complete linkage, H-clu-a — hierarchical clustering algorithm with average linkage, DP-Q1, DP-Q2, DP-Q3 and DP-Q4 $(\Delta)$ - dynamic programming algorithm with scoring function Q1, Q2, Q3 and Q4 $(\Delta)$. In Sec. 6.1, we first define the performance criteria for comparing results of applying different algorithms. Then, we describe 


\section{A. Polanski et al.}

two groups of computational experiments, artificially created data and proteomic mass spectral data, and we report the results of comparisons of different methods for setting initial conditions for EM iterations.

\subsection{Performance criteria}

Performance criteria for evaluating the results of parameter estimation algorithms are based on the values of the differences between true and estimated parameters or on the values of the log-likelihood functions obtained in EM iterations, averaged over repeated experiments. Since in our constructions of the quality criteria we aim at making reasonable comparisons of different initialization algorithms for datasets corresponding to different mixtures, we need to introduce additional scalings and ordering of the values of differences or log-likelihoods, as described below.

Difference between values of true and estimated parameters. The first approach to evaluating the results of mixtures parameter estimation algorithms is using a direct criterion given by a scaled difference between the estimated and true values. We use a scaled absolute difference between true and estimated locations of components, averaged over all components. The scaling is aimed at making the distribution of errors invariant with respect to component widths and weights. The criterion is defined as follows:

$$
D=\frac{1}{K} \sum_{i=1}^{K} \frac{\left|\mu_{i}^{\text {true }}-\mu_{i}^{\text {est }}\right|}{\sigma_{i}^{\text {true }}} \sqrt{N \alpha_{i}^{\text {true }}}
$$

In the above expression, $\mu_{i}^{\text {true }}, \sigma_{i}^{\text {true }}$ and $\alpha_{i}^{\text {true }}$ are true parameters of the analyzed mixture distribution, $K$ is the number of mixture components and $N$ is the sample size. By $\mu_{i}^{\text {est }}$ we understand the value of the estimated mixture component mean closest to $\mu_{i}^{\text {true }}$. Due to the skewness of the distributions of $D$, we use $\log (D)$ as an eventual measure of the quality of parameter estimation.

The value of the quality criterion $\log (D)$ averaged over mixture datasets is denoted as

$$
\operatorname{Avg}[\log (D)]
$$

and further used in reporting results of computational experiments.

Log-likelihoods. The direct criterion defined in the previous section can be used only in the case where the true compositions of the analyzed mixtures are known. Since, we evaluate the methods using data with both known and unknown compositions of mixtures, there is a need for a second approach for evaluating results of mixture parameter estimation algorithms based on values of the log-likelihood functions. The values of the log-likelihoods obtained in the EM iterations can be used for scoring performance of different algorithms in both cases of known or unknown values of true mixture parameters.

In the case of analysing a single dataset, one can use the obtained values of log-likelihoods to order the performance of different algorithms. Higher values of 
log-likelihoods imply a better quality of the solution obtained by using the corresponding algorithm. There are exceptions from the rule "higher likelihood $\rightarrow$ better estimate of mixture parameters" caused by the possible existence of spurious local maximizers [McLachlan and Peel (2000)], but their influence on obscuring the results of evaluations of performances of different algorithms is strongly reduced by the applied constraints on the values of standard deviations of mixture components.

Often, we are not interested in using values of the log-likelihood functions for the analysis of one dataset, but rather for the comparisons involving many datasets. In that case, typically the differences between log-likelihoods obtained for different mixtures (different datasets), are much larger than the differences of log-likelihoods resulting from applying different initialization algorithms for the same data-set. Therefore, the ordering or scaling is used to compensate for this. In this study, we use the criterion similar to that introduced in Karlis and Xekalaki (2003); Biernacki et al. (2003)], defined by the percentage of instances such that the difference between maximum over all obtained log-likelihoods and the $m$ th log-likelihood is lower than $5 \%$ of the range of all log-likelihoods. The value of this criterion, estimated by averaging over repeated computational experiments, is denoted by

$$
\operatorname{Avg}(P) \text {. }
$$

\subsection{Simulated datasets}

The first computational experiment involves analyses of artificially created datasets, which are 10 component Gaussian mixtures, with known values of means, standard deviations and weights of Gaussian components. In the simulated datasets, we are controlling the overlap between Gaussian components due to its strong influence on the results of the fit. Several measures of the degree of overlap between two Gaussian components have been proposed in the literature Sun and Wang (2011)]. Here, we define a simple parameter ov for measuring the degree of overlap between neighboring components,

$$
o v=\exp \left[-\frac{\left|\mu_{i}-\mu_{i+1}\right|}{2 \sqrt{\sigma_{i}^{2}+\sigma_{i+1}^{2}}}\right] .
$$

The parameter ov takes a value equal or close to zero for disjoint components and larger values for components of stronger overlap. The maximal possible value assumed by the overlap parameter is $o v=1$, which occurs in the case where $\mu_{i}=$ $\mu_{i+1}$. The definition of $o v$ in (32) can be interpreted as an adaptation/simplification of the idea of the Bhattacharyya distance. The construction (32) simplifies the overlap definition by the Bhattacharyya distance in the sense that components with equal means, which show maximal possible overlap ov $=1$, can be possibly distinguished based on the Bhattacharyya distance by differences between variances. Despite this simplification, the overlap measure (32) is useful for our analyses, due to the fact that we are not considering mixtures with components of similar means and different variances (claw-like mixtures) McLachlan and Peel (2000)]. 


\section{A. Polanski et al.}

The true parameters of each of the Gaussian mixtures are drawn randomly in each stochastic simulation experiment. The drawing of the parameters of the Gaussian mixtures is designed such that overlaps between neighboring components are constant over one dataset. One stochastic simulation experiment includes three steps:

(1) Randomly draw values of variances and weights of Gaussian components $\sigma_{1}, \ldots, \sigma_{10}$ and $\alpha_{1}, \ldots, \alpha_{10}$.

(2) Define $\mu_{1}=0$ and compute values of means of Gaussian components, $\mu_{2}, \ldots, \mu_{10}$ such that values of the overlapping coefficient 32 between successive components has a given constant value $o v$.

(3) Generate 1,000 independent samples of 10-component Gaussian mixtures with parameters $\alpha_{1}, \ldots, \alpha_{10}, \mu_{1}, \ldots, \mu_{10}$ and $\sigma_{1}, \ldots, \sigma_{10}$.

The differences between individual stochastic simulation experiments involve different methods for drawing weights $\alpha_{1}, \ldots, \alpha_{10}$ and standard deviations $\sigma_{1}, \ldots, \sigma_{10}$ of Gaussian components in the mixtures and different values of the overlapping coefficient $o v$. Four groups of datasets are generated. Each group includes five series of experiments corresponding, respectively, to the following five values of the overlapping coefficient: ov $=0.05,0.1,0.15,0.2$ and 0.25 . Each series corresponds to one value of the overlapping coefficient $o v$ and includes 500 datasets, generated according to steps 1-3. Different groups use different scenarios for generating weights and variances of Gaussian components, described below.

Group 1: Equal mixing proportions. Low variability of standard deviations. In this group, equal values of mixing proportions are assumed, $\alpha_{1}=\alpha_{2}=$ $\cdots=\alpha_{10}=0.1$ and the values of component standard deviations are generated randomly from the uniform distribution $U(0.5,1)$ in each dataset. The values in parentheses give the range of possible changes of standard deviations. This scenario allows only for low (two-fold) variability of the standard deviations of Gaussian components.

Group 2: Equal mixing proportions. High variability of standard deviations. In this group, the values of mixing proportions are assumed to be equal again, $\alpha_{1}=\alpha_{2}=\cdots=\alpha_{10}=0.1$ and the values of component standard deviations are generated randomly from the uniform distribution $U(0.05,1)$. This scenario allows for high (20-fold) variability of the standard deviations of Gaussian components.

Group 3: Different mixing proportions. Low variability of standard deviations. In this group, different values of mixing proportions are assumed, $\alpha_{1}=\frac{1}{55}, \alpha_{2}=\frac{2}{55}, \ldots, \alpha_{10}=\frac{10}{55}$ and the values of component standard deviations are generated randomly from the uniform distribution $U(0.5,1)$, which corresponds to low (2-fold) variability of the standard deviations of Gaussian components.

Group 4: Different mixing proportions. High variability of standard deviations. In this group, different values of mixing proportions are assumed, 
$\alpha_{1}=\frac{1}{55}, \alpha_{2}=\frac{2}{55}, \ldots, \alpha_{10}=\frac{10}{55}$ and the values of component standard deviations are generated randomly from the uniform distribution $U(0.05,1)$, which corresponds to high (20-fold) variability of the standard deviations of Gaussian components.

Comparisons of performances of different algorithms. In the computational experiments, the parameters of the Gaussian mixtures were estimated by using EM iterations, with the initial conditions set by each of the aforementioned algorithms E-Q, H-clu-c, H-clu-a, DP-Q1, DP-Q2, DP-Q3, DP-Q4( $\Delta)$. The performance of the algorithms is evaluated by using quality indices $\operatorname{Avg}[\log (D)] 30$, and $\operatorname{Avg}(P)$ (31). The results, obtained by averaging over 500 datasets in each of the data generation scenarios, are presented in Fig. 1 This figure includes five subplots arranged in columns and rows. The subplots in the two upper rows depict the results of applying the seven initial condition setting algorithms in terms of $\operatorname{Avg}[\log (D)]$. Each of the subplots corresponds to one group of datasets (experiments). In the top-left subplot, showing the results for the experiments with equal weights of components and low variability of the standard deviations of the components, all initialization methods show high performances. In the subplot (second row, left column) corresponding to the experiments with low variability of standard deviations and different component weights, the E-Q method shows lower performance. Other clustering methods (H-clu-c, H-clu-a, DP-Q1, DP-Q2, DP-Q3 and DP-Q4 $(\Delta)$ ) again show quite similar performances, however the differences are bigger than in the previous plot. In the subplots in the right column corresponding to the experiments with high variability of standard deviations of Gaussian components, we can observe stronger diversity between the results of different initialization algorithms. The performance of the E-Q algorithm is high in group 2 (equal component weights), however in group 4 (different component weights) E-Q is the algorithm with the worst performance.

In all the experiment groups, the proposed dynamic programming algorithms DP-Q1, DP-Q2, DP-Q3 and DP-Q4 do not fall behind in $\operatorname{Avg}[\log (D)]$ with regards to the reference methods, often outperforming them. The cases where the performance of the hierarchical clustering method with average linkage can be slightly higher than the dynamic programming methods are groups 1 and 3 (low variability of standard deviations). For groups 2 and 4 (high variability of standard deviations), there is a strong advantage of the dynamic programming algorithms over the reference methods.

High performance of the equal quantiles algorithm in groups 1 and 2, can be considered as a kind of artifact. It does not follow from the high capability of the algorithm to locate positions of components, but rather from the fact that the true values of the component weights coincide with equal quantiles assumed in the algorithm.

We can also make observations concerning the comparisons between variants of the dynamic programming algorithm based on different scoring functions. The performance of the algorithm DP-Q1, based on the scoring function (24) given by 

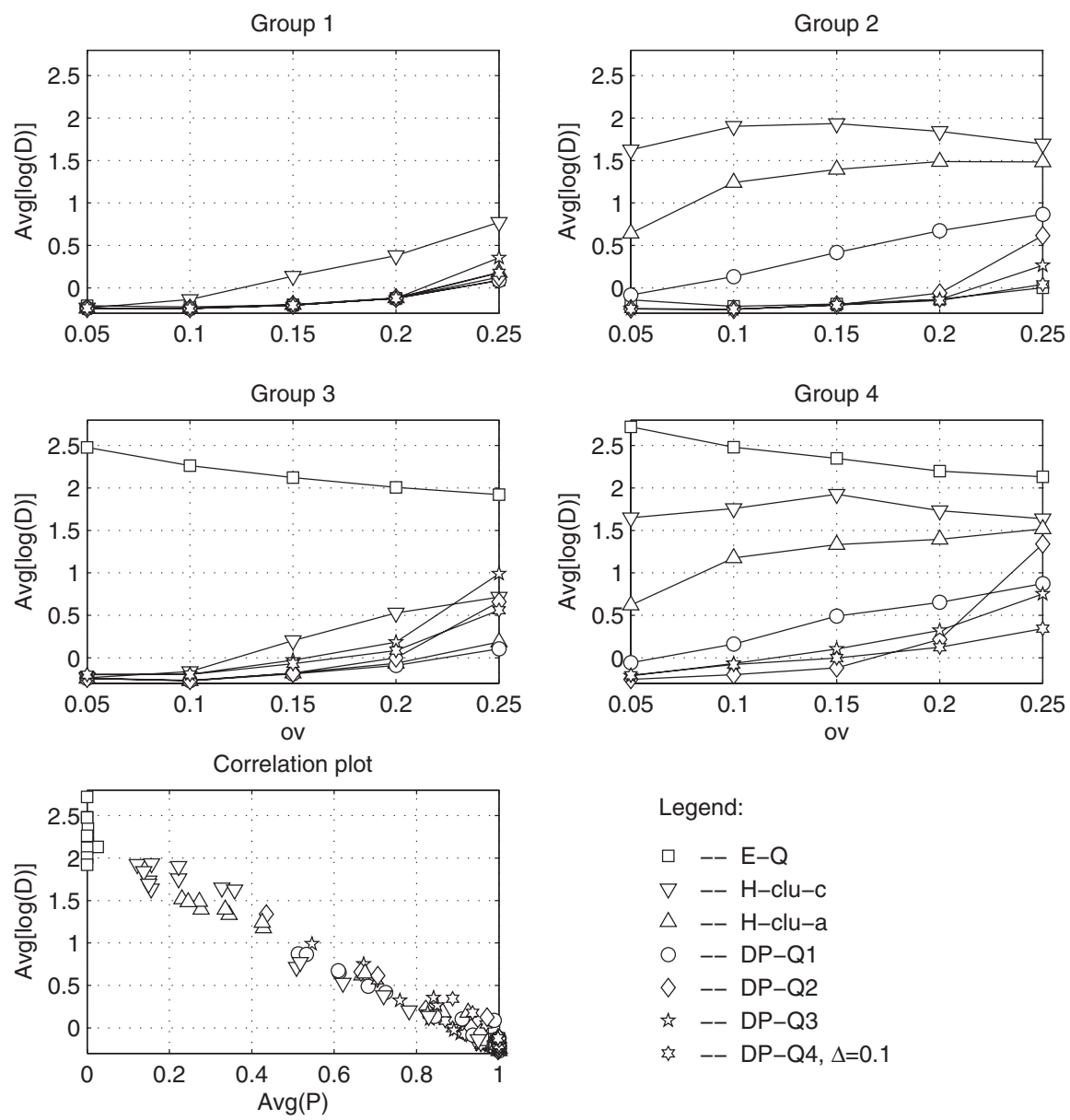

$$
\begin{aligned}
& \text { Legend: } \\
& \square \quad--E-Q \\
& \nabla--\mathrm{H}-\mathrm{clu}-\mathrm{c} \\
& \triangle \text {-- H-clu-a } \\
& \text { ○ -- DP-Q1 } \\
& \diamond--\mathrm{DP}-\mathrm{Q} 2 \\
& \text { is -- DP-Q3 } \\
& \text { \& -- DP-Q4, } \Delta=0.1
\end{aligned}
$$

Fig. 1. Comparisons of results of applying algorithms, E-Q, H-clu-c, H-clu-a, DP-Q1, DP-Q2, DP-Q3 and DP-Q4 $(\Delta)$ for estimating mixture parameters for simulated datasets.

the sample variance, is high in groups 1 and 3 where the variability of component standard deviations is low. However, in groups 2 and 4, where the variability of component standard deviations strongly increases, DP-Q1 exhibits worse performance compared to other variants of the dynamic programming method. This is consistent with the tendency for the dynamic programming partition with the scoring function Q1 to incorrectly merge narrow components. The performance of the algorithm DP-Q2 is high for low values of the overlap coefficient ov, but strongly decreases with the increase of ov. The performance of the algorithm DP-Q4 $(\Delta)$, $\Delta=0.1$ is better than the performance of DP-Q3.

In the bottom-left subplot, we show a scatterplot of the values of indices $\operatorname{Avg}[\log (D)]$ (30) versus the values of the probability index $\operatorname{Avg}(P)$ (31). The scatterplot shows a strong, negative correlation between the values of $\operatorname{Avg}[\log (D)]$ and 
$\operatorname{Avg}(P)$, which confirms the potential of the index $\operatorname{Avg}(P)$ to serve as a reasonable estimate and a comparison tool for the performance of different algorithms.

\subsection{Protein spectra dataset}

A proteomic mass spectrum contains information about mass-to-charge $(\mathrm{m} / z)$ values of registered ions, denoted by $x_{n}$, versus their abundances (the number of counts from the ion detector), denoted by $y_{n}, n$ denotes the index of the data point. In real experiments, the dataset consists of more than one spectrum (sample). Each point $x_{n}$ along the $m / z$ axis has a corresponding count $y_{s n}$, where $s$ denotes the index of the sample.

The second computational experiment in our study was the analysis of the proteomic dataset, which included 52 low resolution proteomic mass spectra of human blood plasma Pietrowska et al. (2011)]. This dataset was obtained in a clinical study where the blood samples were collected from a group of 22 head and neck cancer patients and a group of 30 healthy donors. Raw spectra consisted of approximately $45,000 \mathrm{~m} / z$ values, covering the range of 2,000-12,000 Da. Spectral signals were binned with the resolution $1 \mathrm{Da}$ and baselines were removed with the use of the algorithm described in Sauve and Speed [2004]. For further analysis, the data was filtered to the spectral fragments ranging from 2,000-4,120 Da. The choice of the range 2,000 to $4,120 \mathrm{Da}$ was motivated by the fact that this fragment of the $\mathrm{m} / \mathrm{z}$ scale contains several protein and peptide species interesting as potential candidates for biomarkers.

Comparison of performances of different algorithms. Computational experiments on the proteomic dataset involve modeling spectral signals as mixtures of Gaussian components, estimating model parameters by using the EM algorithm and comparing the qualities of models obtained by using different algorithms of setting initial conditions for EM iterations. The quality criterion for comparing different initialization methods was $\operatorname{Avg}(P)$, where averaging is done over all spectral signals in the dataset. Clearly, the criterion $\operatorname{Avg}[\log (D)]$ cannot be used here due to the lack of knowledge on the true parameters of mixture models.

For spectral signals with a large number of components, high variability of standard deviations and strong, variable overlaps between the components, the differences between the performances of different algorithms of the initialization of EM iterations are magnified compared to the simulated dataset. The application of E-Q, H-clu-c, H-clu-a, DP-Q1, DP-Q2, DP-Q3 and DP-Q4 led to large differences between values of log-likelihood functions of models for the proteomic dataset. Since E-Q, H-clu-c, H-clu-a and DP-Q2 exhibit significantly lower performance than DP-Q1, DP-Q3 and DP-Q4, we have confined the set of compared algorithms to those of the highest quality, DP-Q1, DP-Q3 and DP-Q4. We have decomposed each of the spectral signals into Gaussian mixtures. The EM algorithm was initialized with the use of the following five algorithms: DP-Q1, DP-Q3, DP-Q4 $(\Delta=1)$, DP-Q4 $(\Delta=5)$ 

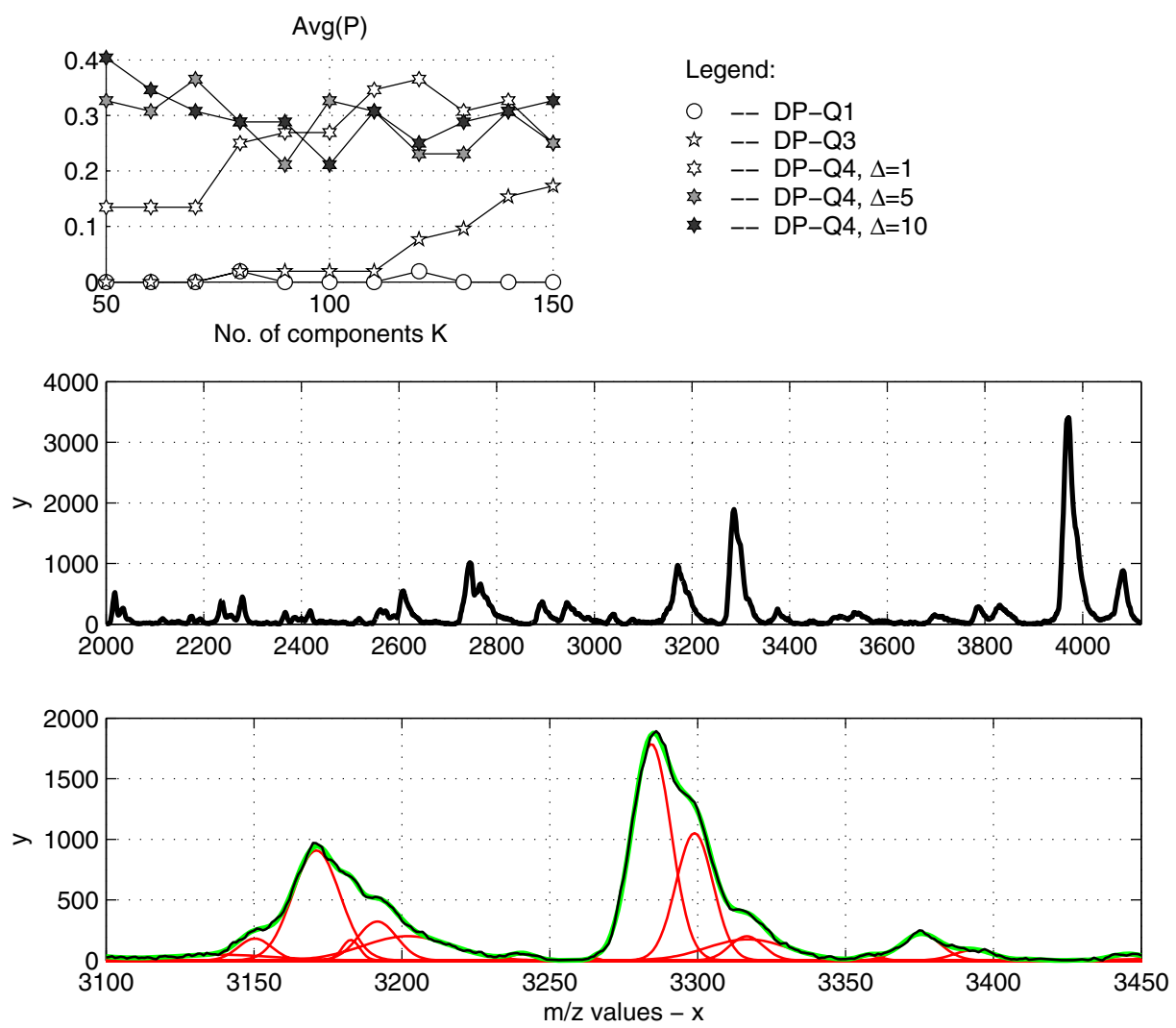

Fig. 2. (Color online) Upper panel: Comparison of performances of algorithms of setting initial conditions DP-Q1, DP-Q3, DP-Q4 $(\Delta=1)$, DP-Q4 $(\Delta=5)$ and DP-Q4 $(\Delta=10)$ for proteomic spectra dataset based on the $\operatorname{Avg}(P)$ index. Middle panel: Spectral signal $y_{n}=y_{1 n}$. Lower panel: A fragment of the spectrum $y_{n}=y_{1 n}$ (black) versus its mixture model (green). The model components are plotted in red.

and DP-Q4 $(\Delta=10)$. The decompositions were computed with numbers of components $K$ ranging from 50 to 150 .

In the upper panel of Fig. 2] we present the plot of values of the index $\operatorname{Avg}(P)$ versus the number of Gaussian components $K$. One can observe that, on average, DP-Q4 shows higher performance than DP-Q1 and DP-Q3. The high performance of DP-Q4 was observed for a wide range of the parameter $\Delta, \Delta=1, \Delta=5, \Delta=10$.

The middle panel in Fig. 2 shows a plot of the spectral signal $y_{n}=y_{1 n}$ (after preprocessing operations of baseline correction and binning) corresponding to sample no 1 within the range 2,000 to 4,120 Da. The spectrum shown in the middle panel in Fig. 2 was decomposed into a mixture of Gaussian components by using of the algorithm DP-Q4 $(\Delta=10)$. The number of components $(K=90)$ was estimated on the basis of the Bayesian information criterion [Schwarz (1978)]. In the lower panel, the plot of a fragment of the spectrum $y_{n}=y_{1 n}$ (black), within the range 
3,100-3,450 Da, is drawn versus its mixture model (green). Gaussian components are plotted in red. One can observe a good fit of the computed mixture model.

\section{Discussion}

Despite the simplicity of the construction of the EM algorithm, the research on its performance and possible improvements is extensive and includes several steps of the algorithm: initialization, stopping conditions, preventing divergence, execution McLachlan and Peel (2000)]. Modifications in each of the above steps interact with each other in the sense of influencing the overall performance of the algorithm. In this study, we have focused on the initialization of EM for certain types of mixture distributions. We have also mentioned some solutions for stopping and preventing divergence. The latter of the above listed issues, concerning modifications of the execution of EM steps for improving its performance, is also worth discussing due to its relations to the topic of our study.

Several papers introduce modifications of the E and $M$ steps of the EM algorithm designed in such a way that searching through the parameter space becomes more intensive, which can help in avoiding local maxima of the likelihood function and make the recursive process more robust against the choice of initial conditions [Zhou and Lange (2010)]. A group of approaches to enhancing the performance of EM iterations involves multiple runs (threads) and repeats of EM steps combined with criteria of selecting between threads Karlis and Xekalaki (2003); Biernacki et al. (2003); O'Hagan et al. (2012)]. The simplest version of short run initiation [Karlis and Xekalaki (2003); Biernacki et al. (2003)] involves generating multiple initial values from random methods and starting EM iterations for the one corresponding to the highest likelihood. Then only one iteration process, namely the one which attained the highest value of the likelihood function, is continued. A recently developed implementation, "burn in EM" [O'Hagan et al. (2012)], involves the continuation of the recursion of multiple threads combined with the gradual filtering to the top performers on the basis of the value of likelihood function. Several ideas of improving the performance of EM iterations are related to modifications of the log-likelihood function corresponding to the Gaussian mixture model. One example of such an approach is the profile likelihood method in Yao 2010. Introducing constraints and/or modifications of the form of the likelihood function both prevent divergence of the iterations and lead to an improvement of the performance of the corresponding variant of the EM algorithm.

Each of the above discussed approaches can be treated as competitive to our algorithm in the sense that it can lead to the improvement of the estimation of the parameters of mixture distributions. We did not present comparisons of our method to the above approaches. However, according to our experience, for the type of data analyzed in this paper (univariate, heteroscedastic, multi-component), precise initialization is more important than possible improvements following from modifications of the execution of EM iterations. We should also mention that 


\section{A. Polanski et al.}

improvements of the EM initialization can be combined with improvements in EM execution to lead to an even better quality of mixture parameter estimation.

In this paper, we assume the known number of mixture components. Analysis of the case of unknown number of components of the mixture model would require repeating computations involving estimation of mixture parameters with known number of components and then choosing the best model on the basis of some criterion, which compromises between quality of fit and the number of components. Most often the BIC criterion would be used [Schwarz (1978)].

Our approach can be potentially applied not only to normal mixtures but also to other mixtures of continuous, symmetric distributions.

\section{Conclusions}

The first conclusion of our study is that the initialization methods based on dynamic programming, DP-Q1, DP-Q2, DP-Q3 and DP-Q4 $(\Delta)$, show an advantage over the reference methods E-Q, H-clu-c and H-clu-a. We have compared the initialization algorithms for a variety of mixture data with the overlap between neighboring components controlled by the parameter (32) and different values of variances and component weights (groups 1-4). This allowed characterizing the dependence of performances of algorithms on parameters of the mixture distributions. The advantage of the dynamic programming initialization methods over the reference methods is highest for heteroscedastic mixture distributions with different mixing proportions.

The second conclusion is that the performance of the dynamic programming partitioning algorithm used for the initialization of EM iterations depends on the scoring function used in the algorithm. We have studied several variants of the algorithm defined by different scoring functions (24)-27). The conclusion coming from these analyses, drawn on the basis of both $\operatorname{Avg}[\log (D)]$ and $\operatorname{Avg}(P)$ criteria, was that for the datasets with different mixing proportions and high variability of standard deviations of components, the most efficient EM initialization method is the dynamic programming algorithm with the scoring function Q4.

We have also applied the dynamic programming partition algorithms DP-Q1, DP-Q3 and DP-Q4 for the initialization of EM iterations for estimating mixture model parameters of a proteomic dataset including 52 low resolution mass spectral signals. A comparison of the values of the $\operatorname{Avg}(P)$ performance criterion lead to the conclusion that the method of the highest efficiency is the dynamic programming partition with the scoring function Q4, consistent with the prior findings on simulated data.

The dynamic programming method with the scoring function Q4 requires the adjustment of the parameter $\Delta$. However, computing decompositions for several values of $\Delta(1,5,10)$ leads to the conclusion that the algorithm shows high performance for a broad range of values of $\Delta$. Adjusting the value of $\Delta$ can be done efficiently and robustly. 
The dynamic programming algorithm applied to the partitioning problem has a quadratic computational complexity with respect to the number of elements of the vector of observations $\mathbf{x}$. Despite its computational load, the advantage of using dynamic programming method for the initialization of the EM algorithm is the quality of the obtained mixture model. In the majority of applications of mixture models, the quality of the model is more important than the computational complexity of the algorithm used for the computations.

\section{Acknowledgments}

This work was financially supported by the National Science Centre, Poland (NCN), grants: 2013/08/M/ST6/00924 (JP), 2016/21/B/ST6/02153 (AP), 2011/01/N/ NZ2/04813 (MM), and 2015/19/B/ST6/01736 (PW and MP). Computations were performed with the use of the infrastructure provided by GeCONiI - Upper Silesian Center for Computational Science and Engineering (The National Center for Research and Development, Poland POIG.02.03.01-24-099/13).

\section{Supplementary materials}

Supplementary materials are Matlab scripts and functions for performing comparisons of partitioning algorithms E-Q, H-clu-c, H-clu-a, DP-Q4 for the data described as Group 4 in Sec. 6.2. Demo computations are started by launching Matlab script partitions-em-demo. One stochastic simulation experiment is performed (including three steps 1-3 listed in Sec. 6.2). Results of computations are shown by plots of partitions and data histograms versus estimated probability density functions. Values of errors $\operatorname{Avg}[\log (D)]$ and likelihoods are also reported. By modifications of the Matlab code other computational scenarios for simulated data can be also easily realized.

Our Matlab scripts and functions are available at the GitHub repository, https://github.com/ZAEDPolSl.

\section{References}

Bellman, R. [1961] "On the approximation of curves by line segments using dynamic programming," ACM Commun. 4(6), 284.

Biernacki, C. [2004] "Initializing EM using the properties of its trajectories in gaussian mixtures," Stat. Comput. 14(3), 267-279.

Biernacki, C., Celeux, G. and Govaert, G. [2003] "Choosing starting values for the EM algorithm for getting the highest likelihood in multivariate gaussian mixture models," Comput. Stat. Data Anal. 41(3-4), 561-575.

Biernacki, C., Celeux, G., Govaert, G. and Langrognet, F. [2006] "Model-based cluster and discriminant analysis with the MIXMOD software," Comput. Stat. Data Anal. 51(2), 587-600.

Bilmes, J. [1998] "A gentle tutorial on the EM algorithm and its application to parameter estimation for gaussian mixture and hidden markov models," Technical Report ICSITR-97-021, University of California Berkeley. 
Chylla, R. [2012] "Metabolite analysis of biological mixtures using adaptable-shape modeling of an online nmr spectral database," J. Comput. Sci. Syst. Biol. 5(1), 51.

Eirola, E. and Lendasse, A. [2013] "Gaussian mixture models for time series modeling, forecasting, and interpolation," in Advances in Intelligent Data Analysis XII, eds. Tucker, A., Hoppner, F., Siebes, A. and Swift, S., Vol. 8207 of Lecture Notes in Computer Science (Springer, Berlin, Heidelberg), pp. 162-173.

Fraiha Machado, A., Bonafonte, A. and Queiroz, M. [2013] "Parametric decomposition of the spectral envelope," IEEE Int. Conf., Acoustics, Speech and Signal Processing $(I C A S S P)$, pp. $571-574$.

Fraley, C. and Raftery, A. [1999] "Mclust: Software for model-based cluster analysis," J. Classif. 16(2), 297-306.

Hastie, T., Tibshirani, R. and Friedman, J. [2009] The Elements of Statistical Learning: Data Mining, Inference, and Prediction, Springer series in statistics (Springer, Berlin).

Hébrail, G., Hugueney, B., Lechevallier, Y. and Rossi, F. [2010] "Exploratory analysis of functional data via clustering and optimal segmentation," Neurocomput. 73(7-9), $1125-1141$.

Ibrahim, M., Lacadie, S. and Ohler, U. [2015] "Jamm: A peak finder for joint analysis of ngs replicates," Bioinformatics 31(1), 48-55.

Ingrassia, S. [2004] "A likelihood-based constrained algorithm for multivariate normal mixture models," Stat. Methods Appl. 13(2) 151-166.

Karlis, D. and Xekalaki, E. [2003] "Choosing initial values for the EM algorithm for finite mixtures," Comput. Stat. Data Anal. 41(34), 577-590.

Kiefer, J. and Wolfowitz, J. [1956] "Consistency of the maximum likelihood estimator in the presence of infinitely many incidental parameters," Ann. Math. Statist. 27(4), 887-906.

Maitra, R. [2009] "Initializing partition-optimization algorithms," IEEE/ACM Trans. Comput. Biol. Bioinform. 6(1), 144-57.

McLachlan, G. and Peel, D. [1999] "The emmix algorithm for the fitting of normal and t-components," J. Stat. Softw. 4(2), 1-14.

McLachlan, G. and Peel, D. [2000] "Finite Mixture Models, Wiley Series in Probability and Statistics (Wiley, New York).

Melnykov, V. and Melnykov, I. [2012] "Initializing the EM algorithm in gaussian mixture models with an unknown number of components," Comput. Stat. Data Anal. 56(6), 1381-1395.

O'Hagan, A., Murphy, T. and Gormley, I. [2012] "Computational aspects of fitting mixture models via the expectation-maximization algorithm," Comput. Stat. Data Anal. 56(12), 3843-3864.

Pietrowska, M., Polanska, J., Walaszczyk, A., Wygoda, A., Rutkowski, T., Skladowski, K., Marczak, L., Stobiecki, M., Marczyk, M., Polanski, A. and Widlak, P. [2011] "Association between plasma proteome profiles analysed by mass spectrometry, a lymphocytebased dna-break repair assay and radiotherapy-induced acute mucosal reaction in head and neck cancer patients," Int. J. Radiat. Biol. 87(7), 711-9.

Polanski, A., Marczyk, M., Pietrowska, M., Widlak, P. and Polanska, J. [2015] "Signal partitioning algorithm for highly efficient gaussian mixture modeling in mass spectrometry," PLOS ONE 10(7), e0134256.

Richardson, S. and Green, P. [1997] "On bayesian analysis of mixtures with an unknown number of components (with discussion)," J. R. Stat. Soc. B 59(4), 731-792.

Sauve, A. and Speed, T. [2004] "Normalization, baseline correction and alignment of high-throughput mass spectrometry data," in Proc. GENSIPS 2004, Workshop on 
Genomic Signal Processing and Statistics, Baltimore, Maryland, USA, May 26-27, 2004, CD-ROM.

Schwarz, G. [1978] "Estimating the dimension of a model," Ann. Statist. 6(2), 461-464.

Sun, H. and Wang, S. [2011] "Measuring the component overlapping in the gaussian mixture model," Data Min. Knowl. Discov. 23(3), 479-502.

Yao, W. [2010] "A profile likelihood method for normal mixture with unequal variance," J. Stat. Plan. Inference 140(7), 2089-2098.

Zhou, H. and Lange, K. L. [2010] "On the bumpy road to the dominant mode," Scand. Stat. Theory Appl. 37(4), 612-631. 